\title{
Separation of sets and Wolfe duality
}

\author{
F. Giannessi - G. Mastroeni
}

Published online: 8 February 2009

C) Springer Science+Business Media, LLC. 2009

\section{Erratum to: J Glob Optim (2008) 42:401-412 DOI 10.1007/s10898-008-9301-2}

Professor Johannes Jahn and his student Christoph Said have pointed out that the proof of Theorem 4.4 of the paper "Separation of Sets and Wolfe Duality, J. Glob. Optim. (2008) 42:401-412" is not correct. Here we give the corrected version.

Theorem 4.4 Let $\bar{x}$ be a global minimum point to (1) and let $A \subseteq M$ be such that there exists $\bar{\lambda} \in D^{*}$ with $(\bar{x}, \bar{\lambda}) \in A$ and $\langle\bar{\lambda}, g(\bar{x})\rangle \leq 0$. Then

$$
f(\bar{x})=\max _{(x, \lambda) \in A} L(x ; \lambda)
$$

Proof From (4) it follows that

$$
\inf _{x \in X} L(x ; \lambda) \leq \inf _{x \in R} f(x)=f(\bar{x}), \quad \forall \lambda \in D^{*},
$$

which implies

$$
L(x ; \lambda) \leq f(\bar{x}), \quad \forall(x, \lambda) \in M .
$$

Since $\bar{x} \in R$ and $\langle\bar{\lambda}, g(\bar{x})\rangle \leq 0$, then the complementarity condition $\langle\bar{\lambda}, g(\bar{x})\rangle=0$ is fulfilled. It follows that

$$
L(\bar{x} ; \bar{\lambda})=f(\bar{x})
$$

which completes the proof.

The online version of the original article can be found under doi:10.1007/s10898-008-9301-2.

F. Giannessi · G. Mastroeni $(\varangle)$

Department of Mathematics, University of Pisa, L.go B. Pontecorvo, 5, 56127 Pisa, Italy e-mail: mastroen@dm.unipi.it

F. Giannessi

e-mail: gianness@dm.unipi.it 
Corollary 4.1 Let $X$ be an open set in $\mathbb{R}^{n}, \bar{x}$ be a global minimum point to (1) and $A:=$ $\left\{(x, \lambda) \in X \times D^{*}: \nabla_{x} L(x ; \lambda)=0,\langle\lambda, g(x)\rangle \leq 0\right\}$. Suppose that the function $(f,-g)$ is a differentiable $\left(\mathbb{R}_{+} \times D\right)$-function on $X$ and that (19) is fulfilled. Then

$$
f(\bar{x})=\max _{(x, \lambda) \in A} f(x) .
$$

Proof Since $(f,-g)$ is a differentiable $\left(\mathbb{R}_{+} \times D\right)$-function then it is also $\left(\mathbb{R}_{+} \times D\right)$ - convexlike on $X$ so that $\mathcal{E}$ is convex (see Remark 4.1); moreover, $L(x ; \lambda)$ is a differentiable convex function with respect to $x$, for every $\lambda \in D^{*}$. From Theorem 4.3 , it follows that there exists $\bar{\lambda} \in D^{*}$ such that

$$
f(\bar{x})=\inf _{x \in X} L(x ; \bar{\lambda}),
$$

or, equivalently,

$$
f(\bar{x}) \leq f(x)-\langle\bar{\lambda}, g(x)\rangle, \quad \forall x \in X .
$$

Computing (41) for $x:=\bar{x}$, we obtain

$$
-\langle\bar{\lambda}, g(\bar{x})\rangle \geq 0 .
$$

From (41), it follows the inequality

$$
L(x ; \bar{\lambda}) \geq L(\bar{x}, \bar{\lambda}), \quad \forall x \in X,
$$

which implies that $(\bar{x}, \bar{\lambda}) \in A \subseteq M$. Since

$$
L(x ; \lambda) \geq f(x), \quad \forall(x, \lambda) \in A,
$$

then, by Theorem 4.4, we have that

$$
f(\bar{x})=\max _{(x, \lambda) \in A} L(x ; \lambda) \geq \max _{(x, \lambda) \in A} f(x),
$$

which leads to (40).

Acknowledgments We want to express our deep gratitude to Professor Jahn and Christoph Said whose remarks allowed us to correct and improve the statements and the proofs of Theorem 4.4 and Corollary 4.1. 